\title{
Learning sets for simple concept identification and reversal shifts
}

\author{
LORRAINE A. LOW, FREDERICK GRONBERG, and BERNICE SHERLING \\ Framingham State College, Framingham, Massachusetts 01701
}

\begin{abstract}
Age differences in learning sets for simple identification tasks and reversal tasks were studied. Three different problems, each consisting of the initial task (IT) followed by the reversal task (RT) were presented to kindergartners and first graders. Half of the subjects received one order of presentation of the specific problems and the other half received a different order. There was a decrease in number of trials to criterion over successive problems, thus indicating a learning set. Kindergartners did more poorly on RT than IT, and first graders did more poorly on IT than RT. For the first graders, the learning set was independent of order of presentation but for the kindergartners learning set was present only with one order. These results were interpreted as reflecting age differences in the elicitation of common mediators.
\end{abstract}

The mediating response plays a central role in a number of theoretical accounts of conceptual development (Goss, 1961; Kendler \& Kendler, 1962; Kendler, 1972). It is hypothesized that in a concept identification task, older children learn a mediating response to the relevant dimension as well as the required instrumental response to the specific stimulus. Younger children acquire only the latter response. One prediction which has received experimental support (Kendler \& Kendler, 1959; Kendler, Kendler, \& Wells, 1960 ) is that reversal shifts requiring the same mediator, but an interfering instrumental response, are learned more rapidly than nonreversal shifts for children who mediate. Children who have not reached the stage of mediational representation do better on nonreversal shifts than reversal shifts. Differences between performance on the original learning and reversal transfer should similarly reflect the presence of mediators and therefore be sensitive to age differences. One aim of the present study was to provide comparisons of performance on the initial task (IT) and reversal task (RT) for different ages.

A further aim of the present study was to determine whether a learning set could be established over a series of concept identification and reversal learning tasks. For IT, the specific relevant dimension varied from problem to problem, but the common solution was the relevance of the novel dimension. For RT, it was choice of the opposite attribute of the same dimension, e.g., large for IT, then small for RT. Therefore, in addition to the specific mediators, a common mediator such as the new one for IT or the opposite one for RT could be elicited

This paper is sponsored by Conan Kornetsky, who takes full editorial responsibility for it. Frederick Gronberg is now at editorial responsibility for it. Frederick Gronberg is now at
Y.M.C.A., Framingham, Massachusetts. The authors wish to thank J. Kaplan, Principal of Devotion School, Brookline, Massachusetts, and William Matthews, Principal of Potter Road School, Framingham, Massachusetts and their staff. The authors also wish to thank Diane Trompoulas and Mindy Klein for their help in conducting the experiment.

Bernice Sherling is now at Mercy College, Detroit, Michigan. in the solution of subsequent problems. If the presence of this common mediator is age-related; a learning set for these problems would be more likely to occur for the first graders than for the kindergartners.

\section{METHOD}

\section{Subjects}

The subjects were 28 kindergartners (ages 5-6) and 28 first graders (ages 6-7) obtained from the local public elementary schools. Seven from each grade were randomly assigned to each of the four conditions.

\section{Stimulus Materials}

The stimuli were shapes of objects such as a chair, a bottle, an umbrella, cut from colored paper and pasted on a $7 \times 9$ in. posterboard. Each set of stimuli consisted of two different shapes and two different colors. The particular shapes and colors were different from set to set and were irrelevant to the solution of the problems. Each set contained one dimension which differed for each problem and was relevant to the solution of the problem. The dimensions were size (large or small), orientation (crooked or straight) and wholeness (broken or unbroken). The large stimuli were 6 in. in length and the small stimuli, 3 in. The stimuli which were labeled crooked were at an angle of $45 \mathrm{deg}$ from the vertical. The stimuli which were labeled broken were cut in half horizontally, and a $1 / 4$-in. space separated each half. Each set, therefore, contained eight stimuli, each of two colors, two sahpes, and two levels of relevant dimension. There were two sets, A and B, for each problem. Both sets contained the same relevant dimension but differed in color and shape. The IT and RT constituted a specific problem.

\section{Procedure}

The subjects in each age group were randomly assigned to one of four conditions. The first treatment variable was stimulus-change (SC) and stimulus-no-change (SNC). The subjects in the SC condition were given Set A on IT and Set B on $\mathrm{RT}$. In the SNC condition, the subjects were given Set A on both IT and RT. The second treatment variable was the order of presentation of the problems (order). In Order 1, the problem with the large-small relevant dimension (LS) was presented first, then crooked-straight (CS), and unbroken-broken (UB) was presented last. In Order 2, Problems UB, LS, and CS were presented first, second, and third, respectively. The age, the order, and SC-SNC, constituted the between-subject variables 

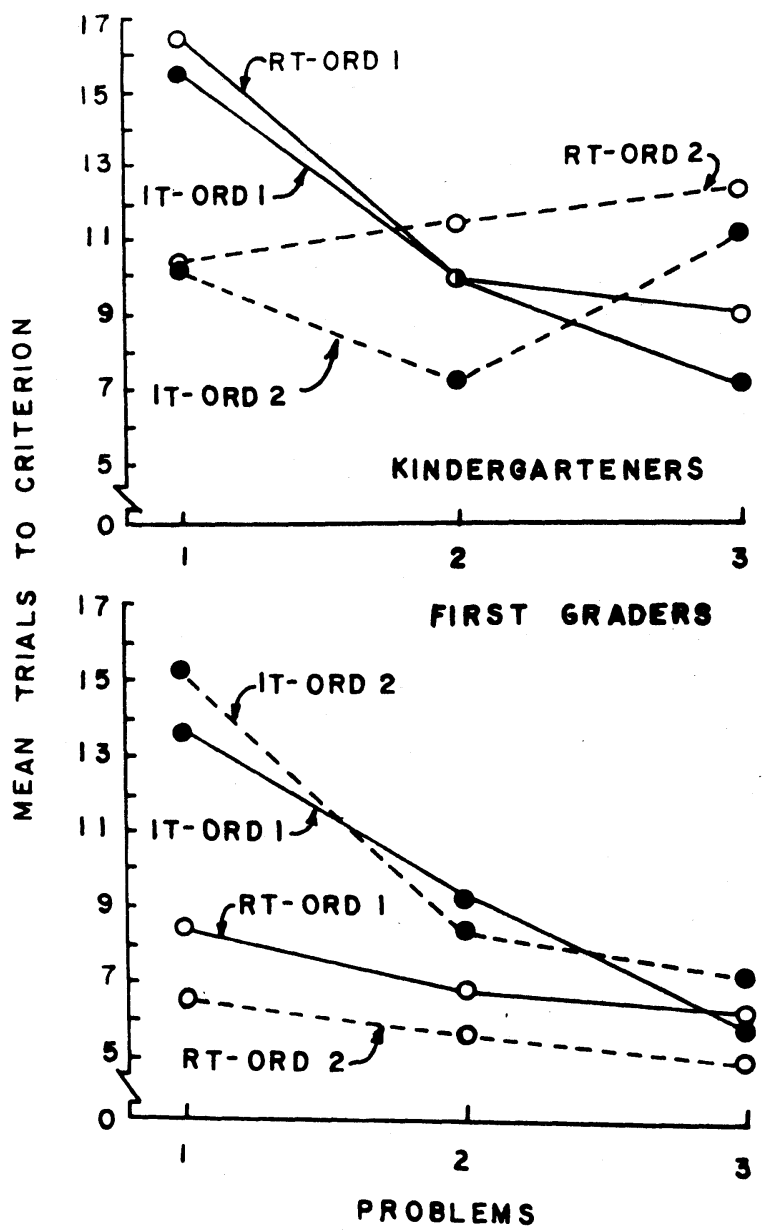

Figure 1. Number of trials to criterion on IT and RT on successive problems as a function of age and order of presentation.

and the three problems (problems) and IT, RT were the within-subject variables.

The subjects were tested individually. The stimuli in a particular set were presented in pairs with a positive and negative value of the relevant dimension present in each pair. One attribute of the relevant dimension was positive for IT, and the opposite was positive for RT, e.g., large, then small. The subject was given three pretraining trials in which pairs drawn from each of the three problems was presented. They were asked to describe how the stimuli differed. The experimenter assisted the subject in verbalizing all the dimensions which the subject omitted. In acquisition and transfer the subject was required to point to the stimulus containing the positive attribute and was then informed of the correctness of his response.

After each task, the subject was told that a new game would follow but that it was to be played the same way.

All subjects were required to attain a criterion of five consecutive correct responses on each task. The IT for a problem was followed immediately with the RT for that problem.

\section{RESULTS}

The score for each subject was the number of trials to reach criterion on each of the six tasks. A mixed analysis of variance was performed on the data with age, SC,
SNC and order constituting the 2 by 2 by 2 between-subject factorial design, problems and IT, RT, the within-subject design. The means of these scores on IT and RT over the three successive problems (Problems 1,2 , and 3) for both kindergartners and first graders is presented in Figure 1 . Age was statistically significant $[F(1,48)=10.75, p<.01]$. The mean number of trials to criterion for kindergartners and first graders were 11.02 and 8.33, respectively. The IT, RT differences were statistically significant $[F(1,48)=4.64, p<.05]$ as well as the Age by IT, RT interaction $[\mathrm{F}(1,48)=20.41$, $\mathrm{p}<.001$ ]. It is evident from inspection of Figure 1 that the kindergartners did more poorly on RT than IT and the first graders did more poorly on IT than RT. It is also evident from Figure 1 that the first graders showed better performance on RT for Problem 1 than IT for Problem 2 with Order 1, and better performance on RT for Problem 1 than IF for Problem 3 with Order 2. Therefore, the better performance of the first graders on RT than on IT cannot be attributable to a general improvement over problems alone.

The difference associated with problems was statistically significant $[F(2,96)=26.67, \mathrm{p}<.001]$. The means for Problems 1, 2, and 3 were 11.92, 8.79, and 8.32 , respectively. The linear component was also statistically significant $[F(1,96)=45.17, p<.001]$. There was a general improvement of performance over the three problems, indicating a learning set. The problem by Order interaction was statistically significant $[F(2,96)=9.48, p<.001]$, as well as Problem by Order by Age $[F(2,96)=9.23, p<.001]$. It is evident from inspection of Figure 1 that for the kindergartners, the learning set effect was contingent upon order. With Order 1 there was a reduction in number of trials to criterion from Problem 1 to Problem 3, but with Order 2 there was no such trend.

\section{DISCUSSION}

The present findings indicated that kindergartners and first graders can form a learning set in a simple identification task. Savaro and Kolodny (1967) obtained learning sets on initial and reversal tasks for four- and five-year-olds. In that experiment, however, the same stimuli were used from task to task and both reversal and nonreversal shifts were within-subject variables. Therefore, the improvement in performance in that experiment was not independent of learning of specific cues in the solution of the problems. The present results extend the previous ones to include learning sets for concept learning and reversal transfer in situations in which the concepts differ between tasks.

The superior performance of the first graders on RT relative to IT and the poorer performance of the kindergartners on RT relative to IT are consistent with the findings of Brown and Scott (1972) that IT, RT differences are age related. These results and the findings of a more general learning set for the first graders, independent of order of presentation provide evidence for a mediational view. The common solution for IT over the three problems was the recognition that the new dimension was relevant and/or the previously irrelevant dimension remained irrelevant to the solution. This invariance of learning set with problem order would strongly suggest that in 
addition to the mediator necessary for solution of the individual problems, a common mediator such as "the new one" was utilized by that group.

The difference between the type of learning set for the kindergartners and first graders compares with the distinction made by Reese (1963) between learning sets and performance sets. Performance sets are a generalized learning-to-learn whereas learning sets involve the acquisition of solutions common to a number of problems The present findings that improvement in performance over problems was not influenced by the order of occurrence of the particular problems lends support to the view that the first graders were acquiring a learning set.

The present results suggest that learning sets for simple concepts could provide a useful mechanism for the study of age-related mediating mechanisms. The specific reversal task proved simple for the first graders as evidence by the ceiling effect on RT. The presence of new irrelevant dimensions during the reversal shifts or the use of other intradimensional shifts as described by Wolff (1967) might provide a more sensitive index of age differences.

\section{REFERENCES}

Brown, A. L., \& Scott, M. S. Transfer between oddity and relative size concepts: reversal and extradimensional shifts. Journal of Experimental Child Psychology, 1972, 13, 350-367.

Goss, A. E. Verbal mediating responses and concept formation. Psychological Review, 1961, 68, 248-274.

Kendler, H. H., \& Kendler, $T$. S. Vertical and horizontal processes in problem solving. Psychological Review, 1962, 69, $1-16$

Kendler, T. S. An ontogeny of mediational deficiency. Child Development, 1972, 43, 1-17.

Kendler, T. S., \& Kendler, H. H. Reversal and nonreversal shifts in kindergarten children. Journal of Experimental Psychology, $1959,58,56-60$

Kendler, T. S., Kendler, H. H., \& Wells, D. Reversal and nonreversal shifts in nursery school children. Journal of Comparative and Physiological Psychology, 1960, 53, 83-88.

Reese, $H$. W. Discrimination learning set in children. In $L$. P. Lipsitt and C. C. Spiker (Eds.)., Advances in child development and behavior, Vol. 1, New York: Academic Press, 1963.

Savaro, A., \& Kolodny, M. Learning set and shift behavior in children. Journal of Experimental Child Psychology, 1969, 7, $21-30$

Wolff, J. L. Concept-shift and discrimination-reversal learning in humans. Psychological Bulletin, 1967, 68, 369-408.

\title{
Memory search as a function of phonological context
}

\author{
ROBERT B. DICK and LARRY HOCHHAUS \\ oklahoma State University, Stillwater, Oklahoma 74074
}

\begin{abstract}
The 11 subjects memorized variable lists of either one or three spoken consonant-vowel (CV) syllables. Each list (positive set) was followed by a spoken CV test syllable and subject's task was to indicate whether or not the test syllable matched a syllable from the list. Negative test probes were either phonemically distinct from items in the list or were phonemically similar to list items. Reaction time ( $\mathrm{RT}$ ) increased with list length and RT was faster to positive than to negative probes. Additionally, a significantly faster memory scanning rate was observed for phonemically distinct negative probes (53 msec/item) than for negative test probes which were similar to items in the positive set $(81 \mathrm{msec} /$ item $)$. Contrary to earlier research it was concluded that phonological distinctive features have a large effect on memory search RT.
\end{abstract}

Analysis of distinctive features of stimulus displays has occupied several researchers in recent years. Gibson's (1969) recently proposed theory of perceptual learning hypothesized that differentiation in visual displays involves the searching and processing of the distinctive

The research for this paper was supported by Oklahoma State University research funds. This paper is sponsored by Robert F. Stanners who takes full editorial responsibility for it.

Robert Dick is now at National Institute of Mental Health, Regional Office IV, Atlanta, Georgia.

Requests for reprints should be addressed to Larry Hochhaus, Department of Psychology, Oklahoma State University, Stillwater, Oklahoma 74074. features present. Employing a disjunctive reaction time task, Yonas and Gibson (1967) found that a visual distinctive feature (diagonality) common to all positive set items produced greater reduction in latencies with practice than was found when the diagonality feature was present in both positive set and negative set items.

The auditory analog and historical basis of Gibson's visual distinctive features is the phonemic distinctive feature. According to Jakobson, Fant, and Halle (1967), any one language code has a finite set of distinctive features and a finite set of rules for grouping these 